\title{
Biosense and Violence: Progress Toward Violence Prevention Using Syndromic Surveillance
}

\author{
Jennifer Vahora* and Stacey Hoferka \\ Office of Health Protection, Illinois Department of Public Health, Chicago, IL, USA
}

\section{Objective}

Our objective was to measure the incidence and prevalence of intentional violent injury and death using Illinois' syndromic surveillance system.

\section{Introduction}

Violence is now clearly recognized as a public health problem ${ }^{1}$. Intentional injuries ranked among the top six leading causes of death for Illinois residents aged 1-44 in 20132. The Illinois Department of Public Health currently collects data on violent injuries and deaths from emergency medical services reports, death certificates, coroner/ medical examiner reports, law enforcement reports, and crime lab reports. However, syndromic surveillance provides near real-time data on violence-related emergency department visits that would increase the timeliness and quality of data available for public health interventions.

\section{Methods}

We developed violence syndromes using text and ICD-9 codes in the chief complaint and diagnosis code data from Illinois emergency departments. These were defined as weapon-related (gunshots and stabbings) and other physical assaults. Legal interventions and emotional/psychological abuse were excluded from the analysis. The violence syndromes were used to describe the number and distribution of cases of injuries treated at Illinois hospitals in 2014 that were attributable to physical assaults, gunshots, and stabbings. The data was stratified by demographic (gender, race, age group), temporal (since January 2014), and spatial (county, regional CD) characteristics. These results were compared with prior-year data reported through the Emergency Medical Service Data System and the National Vital Statistics System, as an initial estimate of data quality.

\section{Results}

The violence query retrieved 13,179 reports for the year 2014. Of these reports, whites $(60.2 \%)$, females $(51.8 \%)$, and the $25-49$ year old age group (33.8\%) had the majority of incidents. Compared to the syndromic surveillance reports, Emergency Medical Service Data System data contained a lower proportion of females (35.4\%) and whites $(36.5 \%)$, and a higher proportion of incidents in Northeastern Illinois (70.8\% vs. $42.0 \%$ ). There was a similar proportion of 25-49 year olds (36.4\%). Data from the National Vital Statistics System indicated a higher incidence of violent death in whites $(68.1 \%)$ and victims aged 25-49 (43.7\%), and lower incidence in females $(20.4 \%)$, compared to syndromic surveillance reports.

\section{Conclusions}

Results obtained from the syndromic surveillance system were comparable to the data in emergency medical service and violent death reporting system for demographic and spatial characteristics. It was not possible to differentiate between new visits of violence-related injuries and repeat visits to EDs for conditions treated previously, but we assumed the rate of duplicacy was similar across emergency medical service and syndromic surveillance data. We also assumed that morbidity and mortality trends in Illinois would be similar, and this assumption may limit the generalizability of our analysis.
Syndromic surveillance has the potential to provide real-time data to inform timely and data-driven public health responses to violence.

\section{Keywords \\ violence; syndromic surveillance; data quality}

\section{References}

1. Dahlberg L, Mercy J. History of violence as a public health issue. AMA Virtual Mentor. 2009 February; 11(2).

2. CDC Injury-WISQARS. [Internet]. Atlanta: Centers for Disease Control and Prevention; c2015. Fatal Injury Reports, 1999-2013, for National, Regional, and States (RESTRICTED); 2015 January 2015 [cited 2015 September 2]; [about 2 screens]. Available from: http:// webappa.cdc.gov/sasweb/ncipc/dataRestriction_inj.html

\footnotetext{
*Jennifer Vahora

E-mail: jennifer.vahora@illinois.gov
} 